Original article

\title{
Severe hair loss associated with psychotropic drugs in psychiatric inpatients-Data from an observational pharmacovigilance program in German-speaking countries
}

\author{
Katrin Druschky ${ }^{\mathrm{a}, *}$, Stefan Bleich ${ }^{\mathrm{b}}$, Renate Grohmann ${ }^{\mathrm{c}}$, Katharina Burda ${ }^{\mathrm{b}}$, \\ Helge Frieling ${ }^{\mathrm{b}}$, Thomas Hillemacher ${ }^{\mathrm{b}, \mathrm{d}}$, Alexandra Neyazi ${ }^{\mathrm{b}}$, Susanne Stübner ${ }^{\mathrm{e}}$, \\ Sermin Toto ${ }^{\mathrm{b}}$ \\ ${ }^{a}$ Department of Neurology, University of Erlangen-Nuernberg, Erlangen, Germany \\ b Department of Psychiatry, Social Psychiatry and Psychotherapy, Hannover Medical School, Hannover, Germany \\ ${ }^{\mathrm{c}}$ Department of Psychiatry and Psychotherapy, Ludwig Maximilian University of Munich, Munich, Germany \\ d Department of Psychiatry and Psychotherapy, Paracelsus Medical University Nuernberg, Nuernberg, Germany \\ e Department of Psychiatry, Kbo-IAK, Academic Teaching Hospital of the Ludwig-Maximilian University, Haar, Munich, Germany
}

\section{A R T I C L E I N F O}

\section{Article history:}

Received 28 May 2018

Received in revised form 20 August 2018

Accepted 20 August 2018

Available online 5 September 2018

Keywords:

AMSP programme

Severe hair loss

Antipsychotic drugs

Antidepressant drugs

Psychotropic drugs

\begin{abstract}
A B S T R A C T
Background: The study aimed to investigate severe hair loss related to psychotropic drugs (PDs) by using data from the drug safety programme Arzneimittelsicherheit in der Psychiatrie (AMSP).

Methods: Data on PD utilization and reports of severe PD-related hair loss were collected in 83 psychiatric hospitals in Austria, Germany and Switzerland during the period 1993-2013.

Results: Out of 432,215 patients under surveillance, 404,009 patients were treated with PDs for the main indications of depression, schizophrenic disorder, neurosis, mania, and organic psychosis. Severe hair loss related to PD treatment was reported in 43 cases $(0.01 \%)$. The rates of hair loss under antipsychotic drugs were slightly lower than the mean rates of all PDs and antidepressant drugs. Valproic acid was related to the highest risk. In 6 of the 43 cases, hair loss was imputed to multiple drugs, with 4 cases imputed to double drug combinations and 2 cases to triple combinations. Rates of severe hair loss under valproic acid (VPA) and lithium salts were distinctly lower as compared with the overall rates reported in literature. Severe hair loss under PD treatment was reported significantly more often in female patients than in male patients $(\mathrm{p}<0.01)$.

Conclusion: The rate of severe PD-related hair loss was very low in the present survey. The large number of patients included in this multicentre study allows for assessment and comparison of hair loss rates related to different PDs and groups of PDs and provides new and supplementary information on PDrelated hair loss.
\end{abstract}

(c) 2018 Elsevier Masson SAS. All rights reserved.

\section{Introduction}

While the occurrence of psychotropic drug (PD)-associated hair loss is considered a rare or infrequent event [1], when it occurs its psychological and psychosocial effects are significant. There are still no reliable treatments available for hair loss and only few successful management options reported in the literature, including for example, topical and oral corticosteroids and the

\footnotetext{
* Corresponding author at: Department of Neurology, University of ErlangenNuernberg, Schwabachanlage 6, 90451 Erlangen, Germany.

E-mail address: katrin@druschky.de (K. Druschky).
}

sensitizing agents diphenylcyclopropenone and dinitrochlorobenzene as potentially effective treatments for alopecia areata [2].

The currently available information on PD-associated hair loss from the medical literature is rather weak and often limited to case reports [3]. Confirmation of drug-induced hair loss is difficult indeed since evidence in the strict sense requires both resolution after discontinuation and reoccurrence after reexposure, the latter not easily being tolerated by affected individuals. Daily loss of up to 150 hairs is regarded as normal, yet, $25-50 \%$ of the head hair must be lost before hair thinning becomes clinically apparent [4,5]. Hair loss can occur for many reasons, ranging from genetic to environmental factors. Androgenetic hair loss, by far the most common cause of hair loss [6], develops in hereditarily predisposed men and women and may be considered part of the natural ageing process. Non-androgenetic hair 
loss can appear under treatment with various drugs or because of toxins, infections, trauma, stress, autoimmune diseases, malnutrition, and endocrine dysfunctions $[7,8]$. Hair growth essentially comprises two main cyclical phases: the anagen and telogen phases. In the anagen phase, which lasts from several months to a decade, new hair shafts are produced. Approximately $80-90 \%$ of the scalp's hair follicles are in this active stage [9]. Telogen, the resting phase, lasts from 2 to 4 months and results in the shedding of the hair shaft. Drug-induced hair loss can either affect the anagen or the telogen phase. Anagen hair loss is typically seen with chemotherapeutic medications and occurs at the time of toxic exposure in a dose-related manner [3,4]. By contrast, telogen effluvium is based on the premature interruption of growth with an early entry of anagen follicles into the resting phase [10]. The established causative factors of telogen effluvium include: iron deficiency, anemia, hyper- or hypothyroidism, delivery, inadequate diet, oral contraception or its discontinuation, accidental exposure to toxic substances, chronic renal insufficiency, and secondary syphilis $[11,12]$. Causative medications comprise beta-blockers, angiotensinconverting-enzyme inhibitors, anticoagulants, oral contraceptives, antithyroid medications, nonsteroidal anti-inflammatory and uricosuric agents, histamine-2-antagonists, lipid-lowering agents, chemotherapy, anticonvulsants, and psychotropic drugs such as several mood stabilizers, certain antidepressants, and some antipsychotics [7,11-13]. Hair loss related to these medications usually appears a few months after starting the medication and is a reversible phenomenon [1].

In the present report, we will describe hair loss as a severe adverse drug reaction related to psychotropic drugs in the routine treatment of psychiatric inpatients using data from the Arzneimittelsicherheit in der Psychiatrie drug safety programme in psychiatry (AMSP). The rates of severe hair loss associated with antidepressants, antipsychotics, mood stabilizers, and other psychotropic drugs were assessed, along with the underlying psychiatric diseases, dosages administered, patient age and sex, and other known risk factors causing hair loss in the respective cases. The large sample size of over 430,000 patients included in this multicentre study will for the first time enable direct comparisons between different PDs and groups of drugs with respect to their related risks of hair loss.

\section{Methods}

The AMSP project was started at the Psychiatric University Hospital of Munich in October 1993 as a continuous multicentre drug surveillance programme serving to assess severe adverse drug reactions (ADRs) in the routine clinical treatment of psychiatric inpatients. ADRs of basically all different organ systems (e.g., psychic, neurologic, gastrointestinal, dermatologic, cardiovascular, haematologic) are acquired. For AMSP purposes, psychotropic drugs also include neurologic drugs such as anticonvulsants and antiparkinsonian drugs.

\subsection{Severe adverse drug reactions}

An ADR is generally rated as severe for three reasons: if it is potentially life-threatening or seriously endangers the patient's health; if it considerably impairs everyday functioning; or if it requires the patient's transfer to another department providing more intensive care [14]. In addition to these overarching criteria, the AMSP study protocol provides additional, more specific guidelines to better coordinate with individual organ classes [15].

\subsection{Severe drug-related hair loss and risk factors}

Severe hair loss in the present study was determined if the patient and the treating physician ascertained a marked or extreme degree of loss in terms of bundles or bunches when combing, washing, or running the fingers through the hair. Other hallmarks included significant thinning of the head hair with the scalp becoming clearly visible and significant loss of body hair including the eyelashes, eyebrows and pubic hair.

Risk factors of hair loss were ascertained by comprehensive medical history, clinical assessment, and laboratory diagnostics through the attending physicians and documented in the case report files. The influence of the documented risk factors on the assessed APD related hair loss rates was addressed by the detailed analysis of each single case (see 2.4 Probability Ratings).

\subsection{Pharmacovigilance methods}

Psychotropic drug utilization data and reports of PD-related rates of severe hair loss were collected in 83 university, municipal, and state psychiatric hospitals in Austria, Germany and Switzerland during the period 1993-2013. The AMSP's pharmacovigilance methods have been described in detail elsewhere $[14,15]$. In short, pharmaco-epidemiological data are gathered at two established, fixed dates per year and include detailed information on drug prescriptions, daily dosages, and individual patient parameters (age, sex and primary psychiatric diagnosis), along with the total number of patients treated per year and per hospital. Specific information on severe adverse drug reactions under psychotropic drug treatment is collected in a second dataset. Trained psychiatrists, known as 'drug monitors', question the ward psychiatrists on a regular basis (i.e., at least every 2 weeks) about the occurrence of severe ADRs, in an open manner assisted by an item list. ADR cases are documented using standardized questionnaires. The generated reports contain a detailed description of the ADR, history, diagnosis, medication, alternative hypotheses on the causes of the ADR, relevant risk factors, countermeasures taken, course of the ADR thereafter, and previous exposures to the drug. The reported cases are discussed at regional and central case conferences that are attended by the drug monitors, representatives from the national drug regulatory authorities, and drug safety experts from the pharmaceutical industry. Assigned ADR probability ratings and the completed case reports are submitted to the relevant authorities and pharmaceutical companies and are stored in the central surveillance database for further analysis. Psychiatric diagnoses are encoded according to the WHO International Classification of Disease (ICD-10). Patient-related data are kept in anonymous form.

\subsection{Probability ratings}

Probability ratings for drug-related severe hair loss were based on the proposals by Hurwitz and Wade [16] and by Seidl et al. [17] and on the AMSP study guidelines [14]. Accordingly, an ADR is rated as "possible" if the ADR is not known for the drug in question, if the time course or dosage of the drug is unusual, or if alternative explanations are more probable. An ADR is rated as "probable" if the ADR is known for the given drug, the time course and dosage are in accordance with previous experience, and if alternative explanations are less likely. ADRs are rated as "definite" if the criteria of "probable" are fulfilled and if re-exposure to the drug elicits reappearance of the ADR.

A preliminary probability rating of AMSP cases is done by the drug monitor. The cases are first sighted by a senior physician of the hospital and then reviewed in detail by senior members of the AMSP management. Finally, cases are discussed at regional and central case conferences. At the regular central AMSP case conferences, attended by clinicians as well as drug safety experts from the medicines regulatory authorities and from the pharmaceutical industry, probability levels (i.e. "definitely", "probably", 
and "possibly" APD related) are discussed by taking into account all possible influence factors. When a consensus is reached, a final probability rating is done and assigned to the corresponding ADR case.

In this study, we investigated all cases of severe hair loss that were rated as possibly (grade 1 ), probably (grade 2 ) or definitely related to PD treatment (grade 3 ).

\subsection{Adverse event recording}

Since drug combination therapies are very frequent in psychiatric inpatient treatment, the AMSP adverse events are evaluated using two different views: In the overall view, all ADR events or, more exactly, all events with at least one drug imputed to be causally related to it are listed under the heading 'all ADR events'. In the targeted view, events are listed when one single drug alone is imputed to be causally related to it. These event rates are listed under the heading 'ADR events imputed to a single drug'. Accordingly, in the present study, the category 'all events' refers to all patients with PD-related hair loss, whereas the category 'hair loss imputed to a single drug' includes only those cases in which a single PD is considered as possibly, probably or definitely responsible.

\subsection{Statistical methods}

Hair loss events are provided in the absolute number of events and in the percentages of patients treated with a drug along with their 95\% confidence intervals. With regard to the low actual ADR incidence rates and the high number of individual patients exposed, the confidence interval $(\mathrm{CI})$ was calculated according to the exact method and not one of the approximate methods [18]. Statistical calculations were performed by means of chi-square tests and were restricted to comparisons of hair loss rates related to diagnoses, sex, and age, since these variables are assessed in the ADR cases as well as in the total population.

\subsection{Ethical section}

Data were obtained from a completely anonymized data bank, and the subjects are not traceable. Evaluations based on the AMSP data bank were approved by the Ethics Committee of the
University of Munich. This study adhered to the Declaration of Helsinki and its later amendments. The AMSP programme is a continuous observational post-marketing drug surveillance programme and does not interfere with the ongoing clinical treatment of the patients under surveillance.

\section{Results}

Severe hair loss was reported in a total of 43 patients by the participating index wards of the AMSP member clinics between 1993 and 2013. The total number of psychiatric inpatients under surveillance was 432,215 , with 404,009 patients treated with PDs, accounting for an overall hair loss rate of $0.01 \%$.

Table 1 shows the different diagnoses, sex, and age groups of the patients with severe hair loss under PDs in comparison to all patients treated. The most common diagnoses of the patients under surveillance were depression, schizophrenic disorder, neurosis, mania, and organic psychosis. Of the patients suffering from severe hair loss, $81.4 \%$ were female, and the mean age was 42.2 years. Female sex was related to significantly higher rates of hair loss $(\mathrm{p}<0.001)$.

The number of patients with hair loss related to PD subclasses and to individual PDs is summarized in Table 2. The left side of the table includes all events of hair loss (one or more drugs imputed), while those imputed to a single drug are shown on the right side of the table. Due to combined PD treatment in some patients, the total number of patients with hair loss in the category 'all events' was lower than the sum of hair loss patients within PD classes or single drugs imputed. Since the evaluation of single PDs excluded those medications with an insufficiently representative number of exposed patients in the total population (case numbers $<5000$ in the present study), the total number of patients with hair loss in the category 'imputed to a single drug' was higher than the sum of hair loss patients within the group of single drugs imputed.

Within the different groups of PDs, the anticonvulsant drugs (represented by valproic acid and lamotrigine) were related to the highest rate of hair loss in both categories ('all cases' and 'drug imputed alone'). Considering the hair loss rates of individual PDs, the elevated risk was caused almost exclusively by valproic acid, which was related to a fourfold rate of hair loss compared to the overall PD-related rate of hair loss. While the rate for the groups

Table 1

Diagnoses, sex, and age groups of patients with severe hair loss under PDs in comparison to all patients treated.

\begin{tabular}{|c|c|c|c|c|}
\hline & \multicolumn{2}{|c|}{ Pts. with hair loss under PDs } & \multicolumn{2}{|c|}{ All patients treated with PDs } \\
\hline & $\mathrm{n}$ & $\%$ & $\mathrm{n}$ & $\%$ \\
\hline \multicolumn{5}{|l|}{ Diagnosis } \\
\hline Depression & 18 & 41.86 & 132,883 & 32.89 \\
\hline Schizophrenic disorder & 13 & 30.23 & 143,151 & 35.43 \\
\hline Neurosis & 5 & 11.63 & 41,737 & 10.33 \\
\hline Mania & 4 & 9.3 & 11,665 & 2.89 \\
\hline Organic psychosis & 3 & 6.98 & 49,607 & 12.28 \\
\hline \multicolumn{5}{|l|}{ Sex } \\
\hline Male & 8 & 18.6 & 177,352 & 43.9 \\
\hline Female & 35 & 81.4 & 226,657 & $56.1^{*}$ \\
\hline \multicolumn{5}{|l|}{ Age } \\
\hline$\leq 30$ & 10 & 23,26 & 69,157 & 17.12 \\
\hline $31-60$ & 28 & 65,12 & 225,418 & 55.8 \\
\hline$\geq 61$ & 5 & 11,63 & 109,434 & 27.09 \\
\hline All pts. & 43 & & 404,009 & \\
\hline Mean age & 42.21 & & 49.23 & \\
\hline
\end{tabular}

PDs, psychotropic drugs; Pts., patients. $\mathrm{p}<0.001$. 
Table 2

Patients with hair loss related to PD subclasses and to single PDs.

\begin{tabular}{|c|c|c|c|c|c|c|c|}
\hline \multirow[t]{2}{*}{ Imputed drug } & \multirow{2}{*}{$\begin{array}{l}\text { Pts. receiving drug } \\
\mathrm{n}\end{array}$} & \multicolumn{3}{|c|}{ All pts. with hair loss } & \multicolumn{3}{|c|}{ Pts. with hair loss imputed to a single drug } \\
\hline & & $\mathrm{n}$ & $\%$ & $95 \% \mathrm{CI}$ & $\mathrm{n}$ & $\%$ & $95 \% \mathrm{CI}$ \\
\hline All PDs & 404,009 & 43 & 0.011 & $0.01-0.01$ & 37 & 0.009 & $0.01-0.01$ \\
\hline \multicolumn{8}{|l|}{ PD groups } \\
\hline Antiepileptic drugs & 86,468 & 17 & 0.020 & $0.01-0.03$ & 15 & 0.017 & $0.01-0.03$ \\
\hline Antidepressants & 207,623 & 18 & 0.009 & $0.01-0.01$ & 14 & 0.007 & $0.00-0.01$ \\
\hline Antipsychotics & 291,510 & 10 & 0.003 & $0.00-0.01$ & 7 & 0.002 & $0.00-0.00$ \\
\hline Lithium salts & 21,202 & 2 & 0.009 & $0.00-0.03$ & 1 & 0.005 & $0.00-0.03$ \\
\hline \multicolumn{8}{|l|}{ Single PDs } \\
\hline Amitriptyline & 12,904 & 1 & 0.008 & $0.00-0.04$ & & & \\
\hline Aripiprazole & 11,054 & 1 & 0.009 & $0.00-0.05$ & 1 & 0.009 & $0.00-0.05$ \\
\hline Citalopram & 22,822 & 2 & 0.009 & $0.00-0.03$ & 1 & 0.004 & $0.00-0.02$ \\
\hline Doxepin & 12,777 & 2 & 0.016 & $0.00-0.06$ & 2 & 0.016 & $0.00-0.06$ \\
\hline Lamotrigine & 9759 & 1 & 0.010 & $0.00-0.06$ & 1 & 0.010 & $0.00-0.06$ \\
\hline Lithium carbonate & 21,202 & 2 & 0.009 & $0.00-0.03$ & 1 & 0.005 & $0.00-0.03$ \\
\hline Mirtazapine & 51,092 & 2 & 0.004 & $0.00-0.01$ & 1 & 0.002 & $0.00-0.01$ \\
\hline Olanzapin & 47,352 & 4 & 0.008 & $0.00-0.02$ & 2 & 0.004 & $0.00-0.02$ \\
\hline Paroxetine & 9736 & 1 & 0.010 & $0.00-0.06$ & 1 & 0.010 & $0.00-0.06$ \\
\hline Quetiapine & 52,370 & 2 & 0.004 & $0.00-0.01$ & & & \\
\hline Risperidone & 45,215 & 3 & 0.007 & $0.00-0.02$ & 3 & 0.007 & $0.00-0.02$ \\
\hline Sertraline & 16,136 & 2 & 0.012 & $0.00-0.04$ & 2 & 0.012 & $0.00-0.04$ \\
\hline Trazodone & 8640 & 1 & 0.012 & $0.00-0.06$ & 1 & 0.012 & $0.00-0.06$ \\
\hline Valproic acid & 37,854 & 16 & 0.0423 & $0.02-0.07$ & 14 & 0.037 & $0.02-0.06$ \\
\hline Venlafaxine & 34,019 & 7 & 0.020 & $0.01-0.04$ & 5 & 0.015 & $0.00-0.03$ \\
\hline
\end{tabular}

$\mathrm{PD}(\mathrm{s})$, psychotropic drug(s); Pts., patients; CI, confidence interval.

consisting of antidepressants or lithium salts ranked almost equally (approximately $0.01 \%$ in the category 'all cases') with the overall PD-related hair loss rate, the rate for the group of antipsychotics ranked somewhat lower, below half the rate of all PDs. With respect to the hair loss rates of individual PDs, mirtazapine and quetiapine were found to be related to slightly lower risks compared to the mean rate of PD-related hair loss. In the category 'drug imputed alone', no cases were reported for amitriptyline and quetiapine, which also suggested a slightly reduced rate of hair loss in comparison to the other PDs, although respective confidence intervals overlapped considerably. Hair loss rates of venlafaxine and valproic acid ranked higher than the mean in both categories, with valproic acid found to be related to the highest rate among the individual PDs (approximately $0.04 \%$ ).
Psychotropic drug polypharmacy was applied in 34 (79.1\%) of the 43 patients experiencing severe hair loss and in 76.6\% of all PDtreated patients and was probably due to the severity of the diseases in this inpatient collective. In 6 patients with PD-related hair loss (15.0\%), more than 1 drug was considered responsible for hair loss, with 'double imputations' in 4 cases and 'triple imputations' in 2 cases. Two of these 'multiple imputations' included combinations of two ADs, and the other combinations consisted of drugs from different groups (combinations of $\mathrm{AD}+$ valproic acid in two cases and one combination each of $\mathrm{AD}+$ lithium and $\mathrm{AD}+\mathrm{AP}$ in the other cases). Only one of the imputed combinations included a non-psychotic drug, namely, dalteparin sodium, which was blamed for hair loss within a triple combination that included valproic acid and olanzapine.

Table 3

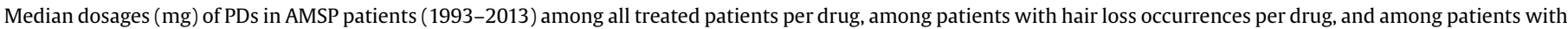
hair loss occurrences per drug imputed alone.

\begin{tabular}{|c|c|c|c|}
\hline PDs & All treated pts. & $\begin{array}{l}\text { Pts. with hair loss as ADR } \\
\text { All cases }\end{array}$ & $\begin{array}{l}\text { Pts. with hair loss as ADR } \\
\text { Drug imputed alone }\end{array}$ \\
\hline Amitriptyline & 100.00 & 25.00 & \\
\hline Aripiprazole & 15.00 & 10.00 & 10.00 \\
\hline Bupropion & 300.00 & 150.00 & 150.00 \\
\hline Citalopram & 20.00 & 40.00 & 40.00 \\
\hline Doxepin & 100.00 & 125.00 & 125.00 \\
\hline Lamotrigine & 150.00 & 50.00 & 50.00 \\
\hline Lithium carbonate & 900.00 & 900.00 & 900.00 \\
\hline Mirtazapine & 30.00 & 37.50 & 45.00 \\
\hline Olanzapin & 15.00 & 12.50 & 10.00 \\
\hline Paroxetine & 30.00 & 30.00 & 30.00 \\
\hline Quetiapine & 250.00 & 412.50 & \\
\hline Risperidone & 3.00 & 2.50 & 2.50 \\
\hline Risperidone depot & 3.57 & 2.68 & 2.68 \\
\hline Sertraline & 100.00 & 75.00 & 75.00 \\
\hline Tranylcypromine & 30.00 & 20.00 & \\
\hline Trazodone & 150.00 & 200.00 & 200.00 \\
\hline Valproic acid & 1200.00 & 1500.00 & 1500.00 \\
\hline Venlafaxine & 150.00 & 150.00 & 150.00 \\
\hline Ziprasidone & 120.00 & 160.00 & 160.00 \\
\hline
\end{tabular}

PDs, psychotropic drugs; Pts., patients; ADR, adverse drug reactions. 
The median PD dosages in patients with severe hair loss and in all the patients treated are presented in Table 3 . The group of hair loss patients was further differentiated into two categories, namely, 'all cases,' in which either the individual drug or the drug in combination with others was causative of the hair loss or to the category 'imputed alone,' in which the individual drug is considered responsible. The median dosages of the PDs administered were within the recommended range for each substance. In 7 of 19 instances, the PD median dosages were somewhat higher in the cases of hair loss, with the largest differences found with quetiapine in the patients in the category 'all cases' and with citalopram in both categories. In the patients experiencing hair loss, quetiapine was administered at a median dosage that was nearly two times higher and citalopram at a median dosage twice as high as that in all the treated patients.

The mean duration between exposure to the drug imputed and the appearance of hair loss was 37.8 days (range 2-129 days, standard deviation 40.1).

Risk factors for hair loss were reported in 10 patients (23.3\%) and are summarized in Table 4 . The most frequent risk factors were thyroid dysfunctions (5 cases, $11.6 \%$ ) and previously experienced hair loss (3 cases, 7.0\%). The use of numerous hair care agents, systemic infection, and chronic cutaneous lupus erythematosus were reported in one case each (2.3\%). Since risk factors were documented for the ADR cases only and are not available for the total population, relative frequencies could not be given for the risk factors.

Discontinuation of the imputed $\operatorname{drug}(\mathrm{s})$ was performed in 31 (72.1\%) of the 43 patients experiencing severe hair loss; dosage reduction was performed in 9 patients (20.9\%); and no changes in dosage were made in the remaining 3 patients (7.0\%). Consultative dermatologic examinations took place in $12(27.9 \%)$ patients.

Clinical follow-up was reported in 39 of the 43 patients and revealed subsiding symptoms in 29 subjects (67.4\%) following discontinuation or dose reduction of the imputed drug(s). Hair loss remained unimproved in the $4(9.3 \%)$ patients with unchanged drug regimen. The drug imputed could not be discontinued in these cases because of the underlying psychiatric disease. Due to early discharge, the further course remained unknown in the remaining 10 cases.

\section{Discussion}

Hair loss rates related to individual psychotropic medications can scarcely be found in the scientific literature. According to dermatological reviews, the incidence rate of drug-related hair loss is generally difficult to determine. First, the possibility of spontaneous hair loss and coincidental use of the drug under suspicion must be considered, and most of the reported cases lack thorough documentation by dermatologists specifically involved in the field of hair pathology [1]. On the other hand, hair loss of lower intensity is difficult to detect and might thus go unreported [4]. So far, the evidence of psychotropic drug-induced hair loss is most convincing for the mood stabilizers, especially lithium and

\section{Table 4}

Known risk factors of patients with severe hair loss under PD treatment.

\begin{tabular}{ll}
\hline Risk factors & number $^{\mathrm{a}, \mathrm{b}}$ \\
\hline Hypothyroidism/Hashimoto-Thyreoiditis (medically adjusted) & 5 \\
Hair loss experienced previously/premature hair loss & 3 \\
Numerous hair care products & 1 \\
Infection and malnutrition & 1 \\
Lupus erythaematodes discoides & 1 \\
\hline
\end{tabular}

PD, psychotropic drug; ADR, adverse drug reaction.

a From a total of 43 pts.

b Multiple counting possible. valproic acid, which both have been associated with a relatively high risk of PD-related hair loss. A 3-year follow-up study of lithium-treated hospitalized patients reported 12 out of 100 patients complaining of hair loss (rate 12\%) [19]. In this survey hair loss was identified in 11 female and 1 male patient. A method of assessment was not described. As risk factors the author identified hypothyroidism in 3 patients, as well as pre-existing eczema and alopecia areata in one patient each. Regrowth of hair after cessation of lithium was reported in 3 patients. In a 1995 review of the literature 643 cases of valproate-induced alopecia were described [20], with a reported frequency of $0.5-12 \%[21,22]$. Decreasing dosages of valproic acid were reported to lower the risk of frequent side effects, including that of hair loss [23]. Just as with our study, most patients with drug-induced hair loss described in the literature were female [24,25]. Women appear to be more concerned about their skin and hair and can therefore be assumed to complain about hair loss more often than men, as is reflected by the results of other studies [7,19]. Furthermore, women are considered more vulnerable to medication-related hypothyroidism, which is an established risk factor for hair loss by itself [24,26].

Regarding antidepressant and antipsychotic medications, current data are mostly limited to case reports, and estimations of their actual incidence rates of hair loss remain difficult to determine [4]. A review of the incidence of hair loss following the administration of tricyclic antidepressants suggested occurrence in approximately $0.01 \%$ of patients [27]. Generally, most antidepressants and a limited number of antipsychotics are associated with a potential, although rare, risk of hair loss [7]. The absolute rates of hair loss are largely unavailable for the individual substances of the respective subgroups, however.

Since in AMSP only severe cases of hair loss are assessed, the drug-related hair loss rates reported here do not include mild and subtle cases and might therefore be expected to be lower compared to previous studies on the general risks of drug-induced hair loss. Indeed, for lithium salts and valproic acid, we identified distinctly lower rates of hair loss as applying to severe cases only. With respect to the tricyclic antidepressants, detected hair loss rates were similar compared to the literature, however, with rates of approximately $0.008 \%$ found for amitriptyline and $0.016 \%$ for doxepin. AMSP characteristics in terms of its observational, naturalistic and retrospective approach, as well as differences between study methodologies in general, complicate the comparison of data across studies, however, and emphasize the benefit of comparisons within a single study comprising a variety of drugs.

The number of antipsychotics that have been associated with hair loss in the literature is small, which suggests a possibly lower risk compared to the risk associated with mood stabilizers and antidepressants; but existing data do not allow direct comparisons of drug groups and individual drugs. According to psychiatric review studies [3], through 1999, only one case of hair loss secondary to antipsychotic treatment had been described in a patient receiving a one-month treatment with haloperidol [28]. Later, reports confirmed the missing documentation of druginduced hair loss with most antipsychotics [7]; up to now, studies on hair loss related to treatment with typical and atypical antipsychotics are very limited [29-31]. From the results of the above-cited studies, haloperidol and chlorpromazine among the typical antipsychotics and olanzapine, risperidone and quetiapine among the atypical antipsychotics are those that have been documented to be associated with hair loss.

The present results confirm the atypical antipsychotics olanzapine, quetiapine and risperidone as agents potentially causing hair loss and reveal a further atypical, aripiprazole, that may possibly induce severe hair loss. Aripiprazole has not yet been associated with hair loss but was imputed in one case of severe hair loss in the present study. 
A new and important aspect of the present results is the direct comparability of data with respect to hair loss rates of various psychotropic drugs and different drug groups. In this sense, we can confirm the outstanding risk of valproic acid among other individual PDs, as previously suggested by literature reviews. More importantly, the present results provide the first indications for a possibly lower risk with the antipsychotic drug group compared to the mean rate of PDs and to the group of antidepressants in terms of severe drug-induced hair loss. Since combinations of various drug groups are frequently used in psychiatric treatment, differences in the related rates of adverse effects provide important clues for physicians confronted with decisions about if and what modifications are to be made. These decisions may further be complicated, however, by the possibility of multiple drugs causing the adverse reaction, as indicated by the multiple imputations in 6 of the 43 cases of severe hair loss examined in the present study.

\subsection{Study limitations}

As mentioned above, evidence of drug-induced hair loss in the narrow sense requires both resolution after discontinuation and reoccurrence after reexposure. Therefore, as in the clear majority of previous studies, definite evidence for the induction of hair loss by the drugs imputed in the present survey is missing. Nevertheless, our results roughly agree with the existing studies in terms of the antidepressant drug-related rates of hair loss, while the lower rates observed for valproic acid and lithium may be related to restriction of the present results to severe cases of hair loss in inpatient treatment, only. The mean period of 38 days between the start of a medication and the onset of drug-induced hair loss is in the lower range but still matches the proposed time interval [7]. Furthermore, in most of the cases reported here, follow-up revealed the improvement of hair loss after discontinuation or reduction of the drug(s) imputed.

The naturalistic design of AMSP very closely reflects the clinical reality of psychiatric inpatient treatment and allows analysis of specific variables in terms of psychiatric diagnoses, gender and age of patients, as well as psychotropic medications and dosages. The different individual risk factors of patients as well as multiple-drug therapy of psychotropic and non-psychotropic origin are inherent factors to be considered, however, and risk factors of individual ADRs cannot be assessed among the entire patient population because of the retrospective and observational study approach. Nevertheless, during the final rating, precise assessments of all factors contributing to each reported ADR as well as reliable judgements on its causality occur in regular meetings of an interdisciplinary team of clinicians and pharmacology experts.

Since the reporting of events depends on clinicians acting as individual drug monitors during routine work, an individual and institutional bias in terms of underreporting is to be considered, depending on the time and motivation of the drug monitor and on the financial means of the participating hospital. Furthermore, no quantitative assessment of hair loss and no regular dermatological consultation was performed in this observational, naturalistic and retrospective study. However, because a severe degree hair loss, as investigated here, is very distracting for the affected patients and is a relatively obvious event, the recorded rates are probably more reliable than those for less obvious ADRs.

The study results reflect psychiatric inpatient treatment, only, and cannot be generalized to outpatient settings.

\subsection{Conclusion}

The rate of severe psychotropic drug-related hair loss in the present inpatient sample of 404,009 patients was found to be very low (0.01\%). Among the individual PDs, valproic acid was associated with the highest rate $(0.04 \%)$, confirming its outstanding risk of drug-related hair loss.

\section{Declaration of interest}

The AMSP drug safety programme is facilitated by nonprofit associations in Germany, Austria and Switzerland. The AMSP programme has been supported with unrestricted educational and research grants since 1993 by the following companies:

Austrian companies: Astra Zeneca Österreich $\mathrm{GmbH}$, Boehringer Ingelheim Austria, Bristol-Myers Squibb GmbH, CSC Pharmaceuticals $\mathrm{GmbH}$, Eli Lilly GmbH, Germania Pharma GmbH, GlaxoSmithKline Pharma GmbH, Janssen-Cilag Pharma GmbH, Lundbeck $\mathrm{GmbH}$, Novartis Pharma GmbH, Pfizer Med Inform, and Wyeth Lederle Pharma GmbH.

German companies: Abbott GmbH \& Co. KG, AstraZeneca GmbH, Aventis Pharma Deutschland GmbH GE-O/R/N, Bayer Vital GmbH, Boehringer Mannheim GmbH, Bristol-Myers-Squibb, Ciba Geigy $\mathrm{GmbH}$, Desitin Arzneimittel GmbH, Duphar Pharma GmbH \& Co. KG, Eisai GmbH, Esparma GmbH Arzneimittel, GlaxoSmithKline Pharma GmbH \& Co. KG, Hoffmann-La Roche AG Medical Affairs, Janssen-Cilag GmbH, Janssen Research Foundation, Knoll Deutschland GmbH, Lilly Deutschland GmbH Niederlassung Bad Homburg, Lundbeck GmbH \& Co. KG, Novartis Pharma GmbH, Nordmark Arzneimittel $\mathrm{GmbH}$, Organon GmbH, Otsuka-Pharma Frankfurt, Pfizer GmbH, Pharmacia \& Upjohn GmbH, Promonta Lundbeck Arzneimittel, Rhone-Poulenc Rohrer, Sanofi-Synthelabo GmbH, Sanofi-Aventis Deutschland, Schering AG, SmithKlineBeecham Pharma GmbH, Solvay Arzneimittel GmbH, Synthelabo Arzneimittel GmbH, Dr. Wilmar Schwabe GmbH \& Co., Thiemann Arzneimittel GmbH, Troponwerke GmbH \& Co. KG, Upjohn GmbH, Wander Pharma GmbH, and Wyeth-Pharma GmbH.

Swiss companies: AHP (Schweiz) AG, AstraZeneca AG, BristolMyers Squibb AG, Desitin Pharma GmbH, Eli Lilly (Suisse) S.A., Essex Chemie AG, GlaxoSmithKline AG, Janssen-Cilag AG, Lundbeck (Suisse) AG, Organon AG, Pfizer AG, Pharmacia, Sanofi-Aventis (Suisse) S.A., Sanofi-Synthelabo SA, Servier SA, SmithKlineBeecham AG, Solvay Pharma AG, Wyeth AHP (Suisse) AG, and Wyeth Pharmaceuticals AG.

Disclaimer statements: K. Druschky, A. Neyazi, S. Stübner: no conflict of interest to be declared; S. Bleich, R. Grohmann, and S. Toto are project managers of the AMSP programme; $S$. Toto has been a member of the advisory board for Otsouka and has received a speaker's honorarium from Janssen-Cilag, Lundbeck, Otsouka and Servier.

\section{Acknowledgements}

The authors are grateful to all participating hospitals and drug monitors for their voluntary and careful collection of data.

\section{References}

[1] Rebora A.. Changes in growth and distribution of hair associated with psychotropic drug use-incidence and mechanisms. CNS Drugs 1997;8:323-34.

[2] Hordinsky M, Donati A. Alopecia areata: an evidence-based treatment update. Am J Clin Dermatol 2014;15(3):231-46, doi:http://dx.doi.org/10.1007/s40257014-0086-4

[3] Gautam M. Alopecia due to psychotropic medications. Ann Pharmacother 1999;33:631-7.

[4] Warnock JK. Psychotropic medication and drug-related alopecia. Psychosomatics 1991;32:149-52.

[5] Blankenship ML. Drugs and alopecia. Australas J Dermatol 1983:24:100-4.

[6] Piraccini BM, Alessandrini A. Androgenetic alopecia. G Ital Dermatol Venereol 2014;149:15-24.

[7] Mercke Y, Sheng H, Khan T, Lippmann S. Hair loss in psychopharmacology. Ann Clin Psychiatry 2000:12:35-42.

[8] Mubki T, Rudnicka L, Olszewska M, Shapiro J. Evaluation and diagnosis of the hair loss patient. J Am Acad Dermatol 2014;71: 415.e411-415.e415. 
[9] Stroud JD. Diagnosis and management of the hair loss patient. Cutis 1987;40:272-6.

[10] Dunagin WG. Clinical toxicity of chemotherapeutic agents: dermatologic toxicity. Semin Oncol 1982:9:14-22.

[11] Sperling LC, Jorizzo JL. Hair disorders in systemic disease. In: Callen JP, Jorizzo JL, editors. Dermatological signs of internal disease. 3rd edn. Philadelphia, USA: Saunders; 2003. p. 315-21.

[12] Brănişteanu DE, Voicu CM, Brănișteanu DC. Alopecia-a challenge for dermatologists. Rev Med Chir Soc Med Nat Iasi 2014;118(1):11-8.

[13] Brodin MB. Drug-related alopecia. Dermatol Clin 1987;5:571-9.

[14] Grohmann R, Engel R, Rüther E, Hippius H. The AMSP drug safety program: methods and global results. Pharmacopsychiatry 2004;37:4-11.

[15] Grohmann R, Engel RR, Möller HJ, Rüther E, van der Velden JW, Stübner S Flupentixol use and adverse reactions in comparison with other common firstand second-generation antipsychotics: data from the AMSP study. Eur Arch Psychiatry Clin Neurosci 2014;264:131-41.

[16] Hurwitz N, Wade OL. Intensive hospital monitoring of adverse reactions to drugs. BMJ 1969;1:531-6.

[17] Seidl LG, Thornton GF, Cluff LE. Epidemiological studies of adverse drug reactions. Am J Public Health Nations Health 1965;55:1170-5.

[18] Vollset SE. Confidence intervals for a binomial proportion. Stat Med 1993;12:809-24, doi:http://dx.doi.org/10.1002/sim.4780120902.

[19] Orwin A. Hair loss following lithium therapy. Br J Dermatol 1983;108:503-4

[20] Pillans PL, Woods DJ. Review: drug-associated alopecia. Int J Dermatol 1995;34:149-58.
[21] Davis R, Peters DH, McTavish D. Valproic acid. A reappraisal of its pharmacological properties and clinical efficacy in epilepsy. Drugs 1994; $47: 332-72$.

[22] McKinney P, Finkenbine R, DeVane CL. Alopecia and mood stabilizer therapy. Ann Clin Psychiatry 1996;8:183-5.

[23] Despland PA. Tolerance to and unwanted effects of valproate sodium. Schweiz Rundsch Med Prax 1994:83:1132-9.

[24] Sarantidis D, Waters B. A review and controlled study of cutaneous conditions associated with lithium carbonate. Br J Psychiatry 1983;143:42-50.

[25] Bork K. Undesirable drug reactions affecting the hair and nails. In: Bork K, editor. Cutaneous side effects of drugs. Philadelphia: WB Saunders; 1988. p. 249.

[26] Kirov G. Thyroid disorders in lithium-treated patients. J Affect Disord 1998:50:33-40.

[27] Wheatley D. Hair loss with antidepressants. Hum Psychopharmacol Clin Exp 1993;8:439-41.

[28] Kubota T, Ishikura T, Jibiki I. Alopecia areata associated with haloperidol. Psychiatry Clin Neurosci 1994:48:579-81.

[29] Leung M, Wrixon K, Remick RA. Olanzapine-induced hair loss. Can J Psychiatry 2002;47:891-2.

[30] McLean RM, Harrison-Woolrych M. Alopecia associated with quetiapine. Int Clin Psychopharmacol 2007;22:117-9.

[31] Kuloglu M, Korkmaz S, Kilic N, Saglam S, Gurok MG, Atmaca M. Olanzapine induced hair loss: a case report. Klinik Psikofarmakol Bülteni 2012;22:362-6. 